barkeit der Gegensätze, bereits vorliegende Studien beispielsweise zur Stellung der Toleranz im Islam zur Kenntnis zu nehmen und auch der Frage nachzugehen, ob es nicht bereits innerhalb der chinesischen Staatsauffassung und/oder in der politischen Praxis bzw. im Alltagsleben Ansätze zur Überwindung dieser Unvereinbarkeiten gibt.

Helwig Schmidt-Glintzer

Mats Lundahl

Peasants und Poverty - a Study of Haiti

Croom Helm, London, 1979, 699 S., £ 19.95

\title{
Giovanni CaPRIO
}

Haiti - wirtschaftliche Entwicklung und periphere Gesellschaftsformation

Haag + Herchen, Frankfurt/M., 1979, 338 S., 48,- DM

Haiti, das ärmste Land der amerikanischen Hemisphäre, ist immer wieder Gegenstand kontroverser Diskussionen, weil seine spezifische Geschichte auch wissenschaftlich eine Herausforderung darstellt: Warum ist ein Land, das sich schon 1804 vom Joch des Kolonialismus befreien konnte, so erschreckend unterentwickelt? Diese scheinbar simple Frage ist denn auch der Ausgangspunkt für die beiden vorzustellenden Studien, die zum gleichen Zeitpunkt erschienen sind und deren Autoren offensichtlich nichts voneinander wußten. Dies ist leicht zu erklären: Lundahl (ein Schwede) hat sich von $1969-76$ wiederholt im Lande aufgehalten und die Realität in sich aufgenommen und analytisch durchdrungen; Caprios Arbeit (eine Frankfurter Dissertation) ist eine Literaturstudie, die, sollten jemals Feldaufenthalte stattgefunden haben, diese nicht erwähnt (er soll allerdings im Lande geboren sein!). Diese Tatsachen allein berechtigen zunächst keineswegs dazu, ein engültiges Urteil über die Qualität der beiden Arbeiten zu fällen, denn so faktenreich und differenziert auch Lundahls Werk ist, es bringt wenig selbst erarbeitetes Primärmaterial (er begründet dies auf S. 12 damit, daß es zunächst einmal gegolten habe, das reichlich vorhandene Material zu systematisieren). Beide Arbeiten sind auch insofern nur bedingt vergleichbar, als sie verschiedene Untersuchungsgegenstände bearbeiten: endogene Prozesse im ländlichen Bereich auf der einen Seite, exogene Faktoren der ökonomischen Entwicklung auf der anderen Seite. Und doch haben beide Studien sich überlappende Sektoren und analytische Schnittflächen, an denen gleichzeitig der unterschiedliche wissenschaftstheoretische Ansatz klar wird. Ubbrigens: beide Autoren sind OOkonomen, aber grundsätzlich auch offen für soziologisch-politikwissenschaftliche Fragestellungen.

Caprio läßt keinen Zweifel daran, was er beschreiben will: die ,,verschiedenen Etappen der Eingliederung der haitianischen Gesellschaft ins Weltsystem“" und die ,,von der externen Durchdringung verursachte wirtschaftliche und gesellschaftliche Umstrukturierung" (S. 17). Er gibt sich als Adept der Dependenztheoretiker und der Schule der ,, strukturellen Hetereogenität" zu erkennen und benutzt eifrig deren Schlagwörter. Dieser Focus gestattet ihm eine Analyse der Außenabhängigkeit der haitianischen Wirtschaft in verschiedenen Zeiträumen und Sektoren: spanische und französische Kolonialzeit, amerikanische Besetzung (1915 - 34); in der Landwirtschaft, im Bergbau und in der Industrie. Besonders eindrücklich wird diese Darstellung, wenn er auf die jüngste Entwicklung der sog. ,,run-away-industries" hinweist (exportorientierte Produktionsfreizonen), die die billige einheimische Arbeitskraft ausnutzen und keine Verkettungseffekte für die Binnenwirtschaft bringen. Caprios Studie mündet denn auch in die zu erwartende Empfehlung, eine ,, autozentrierte Entwicklung " mit teilweiser Abkoppelung vom ,,kapitalistischen Weltmarkt" anzustreben (S. 
294 ff.). Die interne Dynamik der Arbeit verlangt dies - die benutzte Begrifflichkeit ist bis in einzelne Worte eine Blaupause Senghaas'scher Postulate -, aber sie tut dabei so, als handle es sich hierbei um ein $x$-beliebiges Entwicklungsland. Aber Haiti ist nun einmal ein ganz besonderes, ein einmaliges Land dadurch, daß in ihm die Chance zur eigengesteuerten Entwicklunng schon ab 1804 angelegt ist. Notwendigerweise müßte diese Frage stärker in den Mittelpunkt einer solchen Studie Haitis rücken. Caprio vergibt diese Chance, weil er zu stark von allgemeinen Konzepten geblendet ist, und - um es deutlich auszudrücken - ihm offensichtlich auch nicht sehr viel daran liegt, mehr über das zu erfahren, was sich seitdem an sozio-politischer Entwicklung in Haiti abgespielt hat . . . Nur so ist überhaupt erklärbar, daß er zu Sätzen wie dem folgenden fähig ist, die jeden auch nur oberflächlicher Kenner des Landes erstaunen lassen: „,Die auf Haiti bestehende Kultur ist ein Produkt der Metropolen (Frankreich und USA) . . . sie wird benutzt, um die Herrschaft von außen aufrechtzuerhalten" (S. 270).

Man muß zur Studie von Lundahl greifen, um etwas über die Realitäten Haitis zu ergreifen, die Caprio nicht wahrhaben will: nämlich die spezifischen Strukturen und Prozesse endogener Art, die dieses Land erst zu einem Fall sui generis im Spektrum der Entwicklungsländer werden lassen. Lundahls überreichlich dokumentierte Analyse kreist um eine zentrale $\mathrm{Hy}$ pothese: daß die verschiedensten Determinanten zu einem kumulativen Prozeß der Regression der Realeinkommen im Agrarsektor geführt haben (S. 91). Der Rekurs auf Gunnar Myrdal in diesem Zusammenhang ist nicht die einzige Bemühung des Autors, sein Thema in die allgemeine theoretische Diskussion einzuordnen. Diese Rückbindung bezieht sich vornehmlich auf die volkswirtschaftliche Literatur (etwa A. K. Sens ,,Choice of Techniques“, S. 293 -295, und die Produktionsfunktion für Agraroutput, etwa bei Georgescu-Roegen, S. 235-249, mit mathematischen Ableitungen), aber auch auf makrosoziologische Konstrukte wie Geertz' ,,Involution“ (anhand seiner Analysen auf Java), die er als unbrauchbare Parallele ablehnt (S. $250-254)$.

Folgen wir dem Aufbau der Studie Lundahls: Neben einem deskriptiven Kapitel zur Vorstellung der Agrarstruktur Haitis entwickelt er seine zentrale Hypothese auf der Basis dreier Indikatoren: Produktionsstatistiken, Ernährungslage der Bauern, Niedergang der ,,coumbite", einer prä-kooperativen Arbeitsorganisationsform (d. h. das Meßniveau dieser Variablen ist je verschieden).

Im Anschluß daran untersucht er die Agrarstruktur unter den relevanten Determinanten: Vermarktungsstruktur, Bodenerosion, Landreform, die Rolle von Regierung und Verwaltung (einschließlich Gesundheits- und Schulwesen, Agrarkredit, staatliche Finanzpolitik), die Innovations- und Technologieproblematik. Schon an dieser Aufzählung wird deutlich, daß Lundahl das Erkenntnisinteresse der klassischen ökonomischen Analyse verläßt und zu einer politischen Ókonomie des Agrarsektors ausholt, die an Vollständigkeit der Elemente kaum etwas zu wünschen übrigläßt. Aber - und dies muß Caprio erzürnen - die externen Faktoren sind lediglich eine intervenierende Variable, wenn man auch nicht zu dem Schluß kommen kann, daß sie aus der Analyse ausgeblendet sind.

Ohne auf die Ergebnisse der einzelnen Kapitel in diesem Rahmen ausführlich eingehen zu können, läßt sich doch Lundahls Bild der haitischen Realität wie folgt zusammenfassen: die früh erfolgte (wenn auch nicht durchgehende) Landreform hat dazu geführt, daß die politisch führende Schicht des Landes kein aktives Interesse an einer Förderung des Agrarsektors entwickelte; sie preßt lediglich Steuern aus den bäuerlichen Massen und leistet damit einen beträchtlichen Beitrag zum Verarmungsprozeß. Das allgemein kolportierte Bild, daß die Bauern von Latifundisten, Zwischenhändlern und Kredithaien ausgebeutet würden, weist Lundahl zurück; er diagnostiziert eine Wettbewerbssituation ohne „Extraprofite“ in Kre- 
ditgewährung und Vermarktung. Passivität auf seiten der Regierung (ist nicht Steuereintreibung eine relevante Aktivität?) führt zu Passivität auf seiten der Bauern und Stagnation in den Produktionsformen; die demographische Expansion verstärkt die Bodenerosion, reduziert die bäuerlichen Betriebseinheiten und letztlich den Output pro Produzent, was dann zu Unterernährung und Krankheitsanfälligkeit führt. Der kumulative Prozeß nach unten scheint zwingend entwickelt, ein düsteres, fast auswegloses Panorama gezeichnet - das nur noch die Abwanderung in die Städte und die Migration ins Ausland zuläßt -, weil eine Umkehrung dieses Prozesses aussichtslos erscheint. Lundahl zögert nicht, die Regierungen Haitis seit 1804 für diese tragische Entwicklung direkt verantwortlich zu machen. Wird sie jemals in der Lage sein, ihren ,, anti-peasant-bias" zu überwinden?

Vieles an der Analyse des Autors erscheint logisch abgeleitet und faktenreich gestützt, und doch muß sich Zögern einstellen, wo zwei Faktoren möglicherweise unterschätzt worden sein könnten: Wird die bäuerliche Bevölkerung wirklich nicht auf vielfältige Weise ihres Ertrags beraubt (es widerspricht jeder gängigen Erfahrung in vielen anderen Ländern, daß über Vermarktung und Kredit nicht zusätzlich abgeschöpft wird)? Ist es so unerheblich, welche weltmarktorientierten Produktionen spezifischen Einfluß auf die bäuerliche Bevölkerung nahmen? Es ist zu erwarten, daß diese Fragen in der (nun systematisch-empirischen) Literatur zu Haiti aufgegriffen und überprüft werden, denn sie stellen auch eine genuine Herausforderung für die Forschung über den Einzelfall hinaus dar.

Die beiden Autoren haben übrigens inzwischen Kenntnis voneinander genommen. Caprio hat in ,Le Nouveau Monde“ (Port-au-Prince!) vom 5. 8. 79 Lundahls Studie ausführlich besprochen und ihm zu erwartende Defizite vorgeworfen (Unterbewertung der exogenen Faktoren), worauf letzterer (wohl inzwischen in ,,Conjonction“, ebenfalls Port-au-Prince, erschienen) zum Gegenschlag ausholt und ihm u. a. ein ,,marxistisches Prokrustesbett" in der Analyse vorwirft. Wir wollen in dieser Diskussion nicht Position beziehen, dies sei dem aufmerksamen Leser und potentiellen Haitiforscher überlassen. Wir sollten uns freuen, daß zwei anregende Studien zu diesem faszinierenden Land erschienen sind, die - jede auf ihre mehr oder weniger apodiktische Art - gleichwohl einen bedeutenden Erkenntniszuwachs darstellen (als weitere Bereicherung sei zusätzlich hingewiesen auf: Wolf Donner, Haiti-Naturraumpotential und Entwicklung“, Tübingen 1980).

Hans F. Illy

Heinz-Dietrich Ortlieb/Dieter Losch

Südafrika im Umbruch? Wandlungsprobleme einer vielrassigen Gesellschaft Verlag Weltarchiv, 1980

„Always to be right, always to trample forward, and never to doubt, are not these the great qualities with which dullness takes the lead in the world?" - Viele Organisationen, Politiker und Presseorgane scheinen, betrachtet man ihre Stellungnahmen zum Südafrika-Problem, ihren Ehrgeiz darein zu setzen, diese Beobachtung William Thackerays (Vanity Fair, Penguin Books, Seite 421) zu bestätigen. Einseitige und beflissene Verurteilung, radikale Forderungen nach Gleichstellung im gesellschaftlichen und ,full participation“ auch im politischen Bereich sind einfach zu erheben und verleihen den Anschein aufgeschlossener Humanität und moralischer Integrität. Sie kontrastieren vorteilhaft zu der zur Rechtfertigungsideologie ,,verkrampfen“ Afrikaanertums erhobenen Apartheid, durch die versucht worden ist, negative Handlungen positiv zu bemänteln. So verständlich angesichts dieser früher bis zum Äußersten forcierten Rassentrennung und vieler heute noch vorhandener Anstößigkeiten moralische Entrüstung ist, so läßt sich doch auch nicht leugnen, daß die herrschende, ri- 